\title{
The role of the emergency department in the early management of sepsis
}

\author{
Ahed J Alkhatib ${ }^{1,2 *}$ \\ ${ }^{1}$ Department of Legal Medicine, Toxicology and Forensic Medicine, Jordan University Of \\ Science \& Technology, Jordan. \\ ${ }^{2}$ International Mariinskaya Academy, Department of Medicine and Critical Care, Department \\ of Philosophy, Academician Secretary of Department of Sociology. \\ *CorrespondingE-mail: ajalkhatib@just.edu.jo
}

\begin{abstract}
Sepsis is a life-threatening organ failure caused by a dysregulated host response to infection, according to the Society of Critical Care Medicine. The aim of this study was to review the literature about the importance of early management of sepsis in the emergency room. It has been denoted that the emergency department is the first place in the hospital in which the patients with sepsis are diagnosed and treated. Accordingly, the emergency department should be well prepared too early manage septic cases. Taken together, the emergency department plays a crucial role in the early management of sepsis and lowers staying length in hospital, mortality, and health cost.
\end{abstract}

Keywords: sepsis, emergency department, mortality, staying length, health cost.

Received: 03 November, 2021

Accepted: 22 November, 2021

Published: 29 November, 2021

\section{Introduction}

Sepsis is a life-threatening organ failure caused by a dysregulated host response to infection, according to the Society of Critical Care Medicine (SCCM, 2020). Organ dysfunction can result from cellular injury, which is followed by the production of proinflammatory and anti-inflammatory mediators [1]. Sepsis wreaks havoc on every organ system in the body [1]. It causes hypotension in the circulatory system, pulmonary edema in the respiratory system, translocation of bacteria and endotoxins from the gastrointestinal tract into circulation, abrupt renal failure, and encephalopathy, among many other, less well-known effects of sepsis [1]. According to the World Health Organization (WHO), sepsis affects over 30 million individuals worldwide, resulting in approximately 6 million fatalities each year [2]. According to the Centers for Disease Control and Prevention [3], at least 1.7 million adults in the United States experience sepsis each year [3]. The CDC sepsis statistics estimates that around 270,000 of these 1.7 million Americans will die because of sepsis [3]. According to the CDC's sepsis data, septicemia caused 4,378 fatalities in Texas in 2018, making it the ninth greatest cause of death [3].

The prognosis of people with sepsis and septic shock depends on how quickly they are treated. Because the Emergency Department (ED) is likely to be the first point of contact for septic patients, emergency physicians are critical in the early stages of patient management, which include accurate initial diagnosis, resuscitation, and 
antibiotic treatment. Since the Surviving Sepsis Campaign recommendations were released in 2016, multiple studies on various areas of sepsis care have been published, bringing a significant amount of new information to the pathophysiology and treatment of sepsis and septic shock [4].

One of the most difficult tasks for emergency physicians is dealing with septic patients. A dysregulated host response to infection causes sepsis, which is a life-threatening organ failure. Septic shock is a kind of sepsis in which anomalies in the circulatory, cellular, and metabolic systems cause higher mortality [5]. A new algorithm using both the Sequential Organ Failure Assessment (SOFA) and the quick SOFA scores, according to the Sepsis-3 standards, allows for a homogeneous diagnosis of septic patients [5].

The need for early recognition and risk stratification has led to the identification of many prognostic markers that could help the emergency physician implement a more aggressive and effective disease management $[6,7]$. Because sepsis is a time-dependent disease, and the first medical contact of such patients occurs in the Emergency Department (ED) [8,9], the need for early recognition and risk stratification has led to the identification of many prognostic markers that could help the emergency physician implement a more aggressive and effective disease management. Despite this, in-hospital mortality rates in Europe and North America are still high, with rates as high as $40 \%$ [4].

Despite recent breakthroughs in detection and treatment, sepsis remains a leading cause of death in individuals with infections all over the world. During the COVID-19 pandemic, the persistent threat of sepsis to humanity was intensified [10]. However, health disparities thwart efforts to combat sepsis. The World Sepsis Congress 2021 (worldsepsiscongress.org, 2021) focused on how to improve sepsis and COVID-19 prevention, survival, and survivorship. It raises several important problems that clinicians of our generation must address.

In the fight against community-acquired illness and sepsis, emergency departments (EDs) are at the forefront. However, the role of emergency physicians has weakened over time, with interventions hampered by the Sepsis-3 definition, which heavily relies on laboratory test results to confirm organ dysfunction, a lack of reliable sepsis screening and prognostic tools outside the intensive care unit (ICU), and limited ED access to imaging studies to identify organ dysfunction [10].

Technological breakthroughs such as novel sepsis biomarkers may aid doctors in making a more exact diagnosis, but the increased expense, complexity, and turnaround time are pushing sepsis care away from emergency physicians, particularly in resource-constrained settings. There is a pressing need to reintroduce sepsis care to the emergency department. To do so, there is a need for more accurate prognostic algorithms based on routinely obtained clinical or laboratory data. We also need to expand the role of emergency physicians in the treatment of serious infections, such as bloodstream infections [10].

To avoid multi-organ failure and sepsis death, one of the most important concerns is to recognize tissue hypoperfusion immediately using readily available clinical or laboratory indicators [12]. Found that the venous-toarterial carbon dioxide pressure changes and the arteriovenous oxygen content difference ratio ( $\mathrm{Pcv}-\mathrm{aCO} 2 / \mathrm{Ca}-\mathrm{vO} 2)$, a marker for global anaerobic metabolism, is an important predictor of mortality in patients with sepsis or septic shock, in a systematic review and meta-analysis of 13 clinical studies involving 940 patients. A greater $\mathrm{PcvaCO} 2 / \mathrm{Ca}-\mathrm{vO} 2$ ratio was linked to a higher risk of 28 -day death (risk ratio $=1.89,95 \%$ confidence interval $=$ 1.48-2.41) and a higher Sequential Organ Failure Assessment (SOFA) score (standardised mean difference $=1.58$, $95 \%$ confidence interval $=0.88-2.28$ ). This ratio, together with lactic acid clearance and central venous blood oxygen saturation, should be used to guide the effects of early resuscitation treatment in sepsis patients, according to [12]. 
While white cell counts in the total blood picture are frequently used by doctors to make therapeutic decisions, the significance of red cell parameters in sepsis prognosis has not been well investigated in the literature. In a study of 236 patients with sepsis or septic shock, [13] discovered that the red blood cell distribution width (RDW), a parameter reflecting the heterogeneity of red blood cell volume, is an independent predictor of 28-day mortality (hazard ratio $=1.311,95$ percent confidence interval $=1.119-3.011$ ) in sepsis or septic shock patients (hazard ratio $=1.311,95$ percent confidence interval $=1.119-3.011)$. RDW's area under curve (AUC) was equivalent to that of procalcitonin ( 0.727 vs. 0.768 ), 2 suggesting that RDW could be a useful prognostic marker in situations where procalcitonin or other advanced sepsis biomarkers are unavailable. Another fascinating study in this edition, by Gho et al (2019), looked at the use of electrical cardiometry (EC) in pneumonia. Thoracic fluid content (TFC), a measure of water trapped in the lung and pulmonary vasculature, was found to reliably predict death and ICU admissions in $368 \mathrm{ED}$ patients. EC is a non-invasive approach for continually measuring cardiac output at the bedside. For 28-day mortality, the AUC was 0.72 (95 percent confidence interval $=0.71-0.74$ ), and for ICU admission, it was 0.73 (95 percent confidence interval $=0.62-0.82$ ) [13]. Finally, Cheung et al. conducted a local study in which they evaluated and detailed the experience and outcomes of 64 patients with bloodstream infection treated in an emergency medicine ward (EMW) of a rural hospital with limited acute general and intensive care unit (ICU) beds. In-hospital mortality was low (7.8\%), with four of the five fatal cases occurring in a care-limited end-of-life program. Septic workup, identification of the source of infection using bedside ultrasonography, computerized tomography, or echocardiogram if clinically appropriate and empirical antibiotics were all part of the program. [15], demonstrated that a critical care management program for bloodstream infection conducted by emergency physicians in the EMW is feasible without compromising care quality with the right clinical skills and protocols.

These studies showed that there are still numerous ways for emergency physicians to help improve sepsis care. Because of the high mortality and time-sensitive nature of sepsis management, physicians and researchers must not only invest in new resources and novel technology, but also make better use of existent clinical information and resources. There is still a knowledge vacuum about the optimum technique for early identification of individuals at risk in the emergency department. The movement in Hong Kong and many other countries to digitalize ED workflows using structured data and electronic medical records represents a valuable resource for doctors and researchers. The benefits of digitalization include data standards, uniformity, and automation. Predictive models based on a built-in scoring system or artificial intelligence can generate useful digital notifications to help clinicians make better judgments. Digital alerts integrated into ED workflow have been shown to shorten hospital and ICU length of stay for patients with sepsis. With sepsis registries springing up all over the world, it's critical that we keep improving patient care and sepsis survival. It is critical that we bring sepsis care back to the ED at this period in history when we are rebuilding our health systems after the COVID-19 pandemic [16].

Sørensen et al (2021) reported that patients who were diagnosed with sepsis based on their SOFA score had a higher likelihood of having a positive blood culture. Infections with Gram-positive bacteria, pulmonary tract infections, Streptococcus pneumoniae, and polymicrobial infections were also more common in SOFA patients. An elevated risk of death was linked to polymicrobial infection, Staphylococcus aureus, and a group of other species.

Sepsis is a life-threatening illness induced by a dysregulated host response to infection by bacteria, viruses, fungi, or parasites [18]. Sepsis is associated with a high mortality rate, which varies depending on the severity of the infection, and the frequency is increasing globally [5]. A consensus meeting [19] held in 1991 attempted to standardize sepsis diagnosis by defining sepsis as a mix of infection and systemic inflammatory disease (SIRS). Since SIRS focused on an inflammatory response, numerous attempts were made to redefine sepsis. SIRS criteria as a predictive tool for sepsis diagnosis have insufficient specificity and sensitivity, and SIRS criteria necessitate laboratory testing [5]. In 2016, the Sepsis Taskforce (Sepsis-3) redefined sepsis as organ dysfunction induced by a 
dysregulated host response to infection, adopting the Sequential Organ Failure Assessment (SOFA) score in sepsis diagnosis. Quick SOFA (qSOFA), a modified form of the SOFA scoring system, was also used to help bedside doctors identify patients who were at risk of a serious outcome [5]. The lungs, abdomen, and urinary tract are all common sites for bloodstream infections [20]. Gram-negative or Gram-positive bacteria are the most common organisms that cause sepsis. Septic individuals have also been found to have polymicrobial illnesses [18]. Higher mortality has been linked to Enterococcus, Acinetobacter, Pseudomonas species, and Staphylococcus aureus [21, 22]. In addition, infections of the pulmonary system have the highest death rate [23].

\section{Conclusion:}

The early management of sepsis in emergency department is crucial to inappropriate control of sepsis and reducing mortality rates.

\section{DECLARATIONS}

\section{Conflicts of Interest}

The authors declared no potential conflicts of interest with respect to the research, authorship, and/or publication of this article.

\section{REFERENCES}

[1] Society of Critical Care Medicine (2020). Surviving Sepsis Campaign: History. Retrieved from https://www.sccm.org/SurvivingSepsisCampaign/AboutSSC/History.

[2] Burkhart, M. (2021). Improving Sepsis Bundle Compliance in the Emergency Department. The Eleanor Mann School of Nursing Student Works. Retrieved from https://scholarworks.uark.edu/nursstudent/14.

[3] Center for Disease Control and Prevention (2020). Sepsis: data \& reports. Retrieved from https://www.cdc.gov/sepsis/datareports/index.html.

[4] Francesco Gavelli, Luigi Mario Castello, Gian Carlo Avanzi. Management of sepsis and septic shock in the emergency department. Internal and Emergency Medicine, 2021; 16:1649-1661 https://doi.org/10.1007/s11739-02102735-7.

[5] Singer M, Deutschman CS, Seymour CW et al (2016) The third international consensus definitions for sepsis and septic shock (sepsis-3). JAMA 315:801-810. https:// doi. org/ 10. 1001/ jama. 2016.0287.

[6] Lundberg OHM, Lengquist M, Spångfors $M$ et al (2020) Circulating bioactive adrenomedullin as a marker of sepsis, septic shock and critical illness. Crit Care 24:636. https:// doi. org/10. 1186/ s13054-020-03351-1

[7] Castello LM, Gavelli F, Baldrighi M et al (2020) Hypernatremia and moderate-to-severe hyponatremia are independent predictors of mortality in septic patients at emergency department presentation: a sub-group analysis of the need-speed trial. Eur J Intern Med. https://doi.org/10.1016/j.ejim.2020.10.003.

[8] Sivayoham N, Blake LA, Tharimoopantavida SE et al (2020). Treatment variablesassociated with outcome in emergency department patients with suspected sepsis. Ann Intensive Care 10:136. https://doi.org/10.1186/s13613020-00747-8.

[9] Loritz M, Busch H-J, Helbing T, Fink K (2020). Prospective evaluation of the quickSOFA score as a screening for sepsis in the emergency department. Intern Emerg Med 15:685-693. https:// doi.org/10.1007/s11739-019-02258-2.

[10] Kevin KC Hung, Rex PK Lam, CT Lui. Bringing sepsis care back to the emergency department: New prognostic tools and extended role of emergency physicians. Hong Kong Journal of Emergency Medicine, 2021, 28(4) 197-198.

[11] https://www.worldsepsiscongress.org, retrieved in 26/9/2021.

[12] Wang M, Liu T, Niu Z, et al. Utility of venous-to-arterial carbon dioxide changes to arteriovenous oxygen content ratios in the prognosis of severe sepsis and septic shock: a systematic review and meta-analysis. Hong Kong J Emerg Med. Epub ahead of print 1 March 2021. DOI: 10.1177/1024907921994970.

[13] Zhang J, He XH, Yang J, et al. Role of red blood cell distribution width in predicting the prognosis of patients with 
sepsis. Hong Kong J Emerg Med. Epub ahead of print 28 June 2019. DOI: 10.1177/1024907919860647.

[14] Gho K, Woo SH, Lee SM, et al. Predictive and prognostic roles of electrical cardiometry in noninvasive assessments of community-acquired pneumonia patients with dyspnoea. Hong Kong J Emerg Med. Epub ahead of print 4 July 2019. DOI: $10.1177 / 1024907919860643$.

[15] Cheung Y, Ko S, Wong OF, et al. Clinical experience in management of bloodstream infection in emergency medical ward: a preliminary report. Hong Kong J Emerg Med. Epub ahead of print 3 December 2019. DOI: 10.1177/1024907919890495.

[16] Joshi M, Ashrafian H, Arora S, et al. Digital alerting and outcomes in patients with sepsis: systematic review and metaanalysis. J Med Internet Res 2019; 21(12): e15166.

[17] Sørensen, S.T., Abdullah, S.M.O.B., Sørensen, R.H. et al. Microbiological findings in emergency department patients with sepsis identified by the Sepsis-3 criteria: a single-center prospective population-based cohort study. Int J Emerg Med 14, 39 (2021). https://doi.org/10.1186/s12245-021-00360-x.

[18] Martin GS, Mannino DM, Eaton S, Moss M. The epidemiology of sepsis in the United States from 1979 through 2000. N Engl J Med. 2003;348(16):1546-54. https://doi.org/10.1056/NEJMoa022139.

[19] Bone RC, Balk RA, Cerra FB, Dellinger RP, Fein AM, Knaus WA, et al. Definitions for sepsis and organ failure and guidelines for the use of innovative therapies in sepsis. The ACCP/SCCM Consensus Conference Committee. American College of Chest Physicians/Society of Critical Care Medicine. Chest. 1992;101(6):1644-55. https://doi.org/10.1378/chest.101.6.1644.

[20] Tulloch LG, Chan JD, Carlbom DJ, Kelly MJ, Dellit TH, Lynch JB. Epidemiology and microbiology of sepsis syndromes in a university-affiliated urban teaching hospital and level-1 trauma and burn center. J Intensive Care Med. 2017;32(4):264-72. https://doi.org/10.1177/0885066615592851.

[21] Vincent J-L, Rello J, Marshall J, Silva E, Anzueto A, Martin CD, et al. International study of the prevalence and outcomes of infection in intensive care units. JAMA. 2009;302(21):2323-9. https://doi.org/10.1001/jama.2009.1754.

[22] Shorr AF, Tabak YP, Killian AD, Gupta V, Liu LZ, Kollef MH. Healthcare-associated bloodstream infection: a distinct entity? Insights from a large U.S. database. Crit Care Med. 2006;34(10):2588-95. https://doi.org/10.1097/01.CCM.0000239121.09533.09.

[23] Cohen J, Cristofaro P, Carlet J, Opal S. New method of classifying infections in critically ill patients. Crit Care Med. 2004;32(7):1510-26. https://doi.org/10.1097/01.CCM.0000129973.13104.2D. 